\title{
Dissociation of electromyogram and mechanical response in sleep apnoea during propofol anaesthesia
}

\author{
Yaniv Dotan*, Giora Pillar", , Nave Tov*, Ron Oliven*, Uri Steinfeld ${ }^{+}$, Luis Gaitini ${ }^{\S}$, \\ Majed Odeh*, Alan R. Schwartz ${ }^{f}$ and Arie Oliven*
}

ABSTRACT: Pharyngeal collapsibility during sleep is believed to increase due to a decline in dilator muscle activity. However, genioglossus electromyogram (EMG) often increases during apnoeas and hypopnoeas, often without mechanical effect.

17 patients with obstructive sleep apnoea were anaesthetised and evaluated from termination of propofol administration to awakening. Genioglossus EMG, flow and pharyngeal area (pharyngoscopy) were monitored. Prolonged hypopnoeas enabled evaluation of the relationships between genioglossus EMG and mechanical events, before and after awakening. Additional dilator muscle EMGs were recorded and compared to the genioglossus. Electrical stimulation of the genioglossus was used to evaluate possible mechanical dysfunction.

Prolonged hypopnoeas during inspiration before arousal triggered an increase in genioglossus EMG, reaching mean \pm SD $62.2 \pm 32.7 \%$ of maximum. This augmented activity failed to increase flow and pharyngeal area. Awakening resulted in fast pharyngeal enlargement and restoration of unobstructed flow, with marked reduction in genioglossus EMG. Electrical stimulation of the genioglossus under propofol anaesthesia increased the inspiratory pharyngeal area (from $25.1 \pm 28$ to $66.3 \pm 75.5 \mathrm{~mm}^{2} ; \mathrm{p}<0.01$ ) and flow (from $11.5 \pm 6.5$ to $18.6 \pm 9.2 \mathrm{~L} \cdot \mathrm{min}^{-1} ; \mathrm{p}<0.001$ ), indicating adequate mechanical response. All additional dilators increased their inspiratory activity during hypopnoeas.

During propofol anaesthesia, pharyngeal occlusion persists despite large increases in genioglossus EMG, in the presence of a preserved mechanical response to electrical stimulation.

KEYWORDS: Dilator muscles, electromyogram, genioglossus, sleep apnoea, upper airway

$\mathbf{T}$ he human pharynx is characterised by instability and varying levels of collapsibility that depend on the level of consciousness: during wakefulness, reflex activation of upper airway dilator muscles prevents pharyngeal collapse and maintains adequate upper airway patency [1]. During sleep, these protective mechanisms are deranged [2], and the pharyngeal stability of patients with obstructive sleep apnoea (OSA) waxes and wanes [3], moving between "active", relatively stable periods with little or no disturbance to airflow and periods of a "passive" state with increased collapsibility leading to flow limitation up to complete obstruction [4]. Anaesthesia causes a dose-dependent increase in pharyngeal collapsibility [5] resulting in upper airway obstruction in almost every person [6], as described during the state of coma [7].

Pharyngeal instability during sleep is believed to be due primarily to decreased activation of the upper airway dilator muscles during sleep. This assumption is supported by the decline in pharyngeal dilator muscle electromyogram (EMG) activity observed during the onset of sleep [8,9]. However, there is abundant evidence suggesting that this decline does not persist throughout sleep. Pharyngeal obstruction is known to trigger an increasing drive to the respiratory muscles, reflected by increasing inspiratory intrathoracic negative pressure [10] associated with gradual increase in diaphragmatic EMG [11]. In both animals and humans, activation of the upper airway dilator muscles is tightly linked to the inspiratory muscles, both during sleep and anaesthesia, and increases in response to chemostimulation relatively more intensively than activation of the diaphragm [12, 13]. A gradually increasing dilator muscle EMG activity could be documented even in the first few breaths of flow limitation [14]. Accordingly, the increase in dilator muscle activity

\section{AFFILIATIONS}

${ }^{*}$ Dept of Internal Medicine, Bnai-Zion Medical Center, Technion,

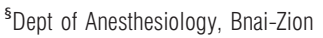
Medical Center, Technion,

"Dept of Pediatrics, Carmel Medical Center, Technion,

\#Sleep Laboratory, Rambam Medical Center, and

${ }^{+}$Dept of Family Medicine, Clalit Health Services, Haifa, Israel.

${ }^{\text {f}}$ Sleep Disorders Center, Johns Hopkins School of Medicine, Baltimore, MD, USA.

CORRESPONDENCE

Y. Dotan

Dept of Internal Medicine

Bnai-Zion Medical Center

47 Golomb Str.

Haifa

31048

Israel

E-mail: yanivdotan@gmail.com

Received:

Sept 142011

Accepted after revision:

March 202012

First published online:

May 032012 
during apnoeas and hypopnoeas would have been expected to restore pharyngeal patency also in OSA patients, even if EMG activity were to decline prior to the apnoeic events and several hypotheses were suggested to explain the failure of dilator muscles to maintain or restore pharyngeal patency during sleep [11]. In a recent study, YounES et al. [15] suggested that dilator muscle activation needs to pass a threshold in order to enlarge the pharynx after the development of even partial obstruction. Although this threshold was often rather low during sleep (a few percent of maximum), arousal usually occurred earlier. According to this explanation, only the low arousal threshold prevents the dilator muscles from reaching the activation threshold needed to become mechanically effective and ameliorate airflow.

In the present study, we hypothesised that progressive dilator muscle activation in response to pharyngeal obstruction also persists during "pharmacological sleep" and that under this condition, which prevents arousal, large increases in dilator muscle activation fail to restore pharyngeal patency. Accordingly, we used propofol anaesthesia to prevent arousal and prolonged flow limitation to stimulate upper airway dilator muscle activity. The relationship between changes in EMG of the genioglossus (GG) and changes in pharyngeal cross-sectional area (CSA) and airflow was compared before and after awakening from propofol anaesthesia. In addition, changes in flow and pharyngeal CSA during augmented GG EMG activity, triggered by prolonged hypopnoea, were compared to changes observed during electrical stimulation of the GG.

\section{METHODS}

\section{Subjects}

17 patients with obstructive sleep apnoea (apnoea/hypopnoea index $>5$ events $\cdot h^{-1}$ ), previously diagnosed in a full sleep study in the Technion Sleep Laboratory (Haifa, Israel), were recruited for this study. Patients with any disease that could pose a risk for anaesthesia, including ischaemic heart disease, any lung disease, severe or uncontrolled hypertension, and body mass index $>35 \mathrm{~kg} \cdot \mathrm{m}^{-2}$, as well as subjects with known side-effects to any previous anaesthesia, were excluded. All studies were performed in the respiratory research laboratory of Bnai-Zion Medical Centre (Haifa). The aims and potential risks of the study were explained, and informed consent was obtained from all subjects. The study was approved by the institutional human investigations review board (Helsinki Committee, Bnai-Zion Medical Center).

\section{Recording procedures}

C4-A1 and C3-A2 electroencephalogram (EEG), ECG and arterial oxygen saturation $\left(\mathrm{Sa}_{1} \mathrm{O}_{2}\right)$ were employed to monitor anaesthesia and recognise arousal. Subjects breathed through a tight-fitting nasal mask and a pneumotachometer connected to a Validyne (Northridge, CA, USA) $\pm 2-\mathrm{cmH}_{2} \mathrm{O}$ pressure transducer, with the mouth carefully and tightly sealed using a chin strap (Respironics, Murrysville, PA, USA) and adhesive tape (Micropore Medical Tape; 3M, St Pauls, MN, USA). The pneumotachometer was connected to a digitised variable pressure source at the inflow port, enabling variation of nasal pressure $(P n)$ of $20--10 \mathrm{cmH}_{2} \mathrm{O}$ (ResMed, San Diego, CA, USA). $P \mathrm{n}$ was monitored with a catheter connected to a side port of the mask. Intrathoracic pressure was measured with an oesophageal balloon catheter (Ackrad Laboratories, Cranford, NJ, USA), used to help recognise upper airway airflow limitation. Analogue to digital acquisition of all parameters was performed at $1,000 \mathrm{~Hz}$ for monitoring and data storage on a digital polygraphic data acquisition system (LabVIEW; National Instruments, Austin, TX, USA).

\section{Electrical stimulation}

Electrical stimulation of the GG was applied via pairs of Tefloncoated, 0.007-inch diameter hook-wire electrodes with bared ends, inserted sublingually, bilaterally, $10-15 \mathrm{~mm}$ deep into the anterior, retromandibular body of the GG, under direct vision, as previously described $[16,17]$. Four to six electrodes were inserted into the GG of each subject. 40-Hz bursts of 2-6 s, with biphasic pulses of $100 \mu$ s width, were applied using a neuromuscular stimulator (Dynex III; Medtronic Inc., Minneapolis, MN, USA). Pharyngoscopic observation and flow monitoring enabled the choice of stimulation intensity that provided the best pharyngeal dilatory response. The intensity of stimulation was limited to levels that were well tolerated during wakefulness in previous and preliminary experiments.

\section{Electromyogram}

GG EMG was recorded in all 17 patients via electrode pairs inserted near to the electrodes used for electrical stimulation. Similar electrodes were inserted into one or two additional upper airway dilator muscles in eight patients: geniohyoid (inserted transcutaneously in the mid-submandibular region, two patients); masseter (inserted transcutaneously directly into the body of the muscle above and anterior to the mandibular angle, four patients); medial pterygoid muscle (inserted transcutaneously below the mandibular angle with the needles directed superiorly adjacent to the internal surface of the mandible, four patients). The masseter and geniohyoid were palpated and adequate needle insertion sites were chosen before anaesthesia, while patients were asked to clench their teeth together forcefully. The site of the medial pterygoid muscle was based on anatomical considerations, as this is the only muscle at the site we used for EMG recording. The GG is also unmistakably visible and palpable close to its insertion site behind the mandible during anaesthesia. In addition, surface submental EMG was recorded in four patients. Raw EMG signals were filtered $(30-1,000 \mathrm{~Hz})$, rectified and processed with leaky integrators with a time constant of $100 \mathrm{~ms}$ to yield a moving-time-averaged EMG envelope, used to measure expiratory (tonic) and peak inspiratory (phasic) activity.

\section{Pharyngoscopy}

A flexible fibre-optic endoscope (Olympus BF-3C40; Tokyo, Japan; 3.3-mm outer diameter), was inserted through an adequately sealed port in the nose mask and positioned above the velopharynx, as previously described [17]. The image was recorded on videotape accompanied by audio explanations, enabling later extraction of the velopharyngeal CSA.

\section{Anaesthesia}

Propofol anaesthesia was delivered by an anaesthesiologist using a loading dose of $2.5 \mathrm{mg} \cdot \mathrm{kg}^{-1}$ and a continuous drip of 6$12 \mathrm{mg} \cdot \mathrm{kg}^{-1} \cdot \mathrm{h}^{-1}$. Using stable $P_{\mathrm{n}}$ levels that enabled breathing without flow limitation, we aimed to maintain the patient under stable anaesthesia that eliminated reaction to pain or to 
movements of the pharyngoscope, while maintaining adequate ventilation, as monitored by the pneumotachometer and pulse oxymetry.

\section{Experimental procedure}

Patients were prepared with EEG and venous access, and placed in the supine position. Following induction of anaesthesia, $P_{n}$ was raised to the level that abolished flow limitation (holding pressure); the pharyngoscope, oesophageal balloon and intramuscular electrodes were positioned, and the mouth was sealed. The site of collapse was determined visually (pharyngoscopy) by gradually lowering $P \mathrm{n}$, and was at the level of the velopharynx in all patients. Thereafter, flow $-P \mathrm{n}$ and $\mathrm{CSA}-\mathrm{Pn}_{\mathrm{n}}$ relationships before and during GG stimulation were determined quasisimultaneously as previously described [17]. With the patient maintained on holding pressure, $P$ n was lowered randomly for a few breaths, encompassing four to six levels associated with inspiratory flow limitation and the level below which airflow ceased and the velopharynx occluded. At each $P_{n}$ level, after the fourth breath, electrical stimulation of GG was performed for two to three consecutive breaths, and after an additional two to three unstimulated breaths, $P \mathrm{n}$ was raised back to the holding pressure, until stable baseline ventilation was observed. After collecting the data (used as part of the data reported in a previous study [17]), propofol administration was stopped. Based on the flow observed at various $P_{n}$ levels, $P_{n}$ was lowered to a stable level of moderate flow limitation with adequate ventilation that could be sustained for prolonged observation. $\mathrm{Sa}_{1} \mathrm{O}_{2}$ was maintained $>95 \%$ with supplemental oxygen whenever needed. All parameters were monitored until wakefulness. A few minutes after awakening, the pharyngoscope and oesophageal balloon catheter were removed, EMG electrodes were left in place, and the patients were maintained on continuous positive airway pressure (CPAP) until fully awake.

After complete recovery from anaesthesia, when the patients were fully alert and cooperative (20-30 min after awakening), maximal awake EMG levels were recorded. To obtain maximal GG, geniohyoid and submental muscle activity, the patients were asked to push their tongue repeatedly as forcefully as possible against the inner surface of the front teeth. Patients with electrodes inserted into the masseter or the medial pterygoid were asked to clench their teeth together as forcefully as possible.

\section{Data analysis}

Airflow, velopharyngeal CSA and EMG activity data of five stable breaths recorded shortly before arousal, and five stable consecutive breaths recorded shortly after arousal, during quiet and regular breathing, under the same level of $P_{n}$, and before removal of the pharyngoscope and the oesophageal balloon, were averaged. EMG activity during expiration was considered as tonic activity, and peak activity during inspiration (i.e. tonic plus phasic activity) is referred to as inspiratory activity. Both were presented as percent of the maximal values obtained during wakefulness. Airflow was measured at the middle, relatively stable portion of the flow-limited breaths, or peak flow when unobstructed flow was restored after awakening. Single pictures of the velopharynx during the end-expiratory pause (in the absence of flow, intrapharyngeal pressure is equal to $P_{n}$ ) and peak inspiration (intrapharyngeal pressure is below $\mathrm{Pn}$ ) were captured from the video movies of the pharyngeal lumen, digitised and stored. The oesophageal pressure tube, marked at regular levels, was used as a landmark in addition to pharyngeal structures for the level at which to measure CSA and as a reference for calculating the CSA in absolute units. The pharyngeal lumen of each digitised frame was outlined manually and calculated digitally using computer software. Flow, CSA and GG-EMG data obtained from the flow $-P \mathrm{n}$ and $\mathrm{CSA}-\mathrm{Pn}$ relationships were used to extract baseline data at the $P$ n level used for continuous flow limitation, as well as the response to electrical stimulation of the GG at the same Pn. ANOVA was used to compare data observed during the baseline, GG stimulation, pre-arousal and post-arousal periods. $\mathrm{p}<0.05$ was considered significant.

\section{RESULTS}

The anthropometric and polysomnographic characteristics of the patients are given in table 1. All patients were male. The group studied had a wide range of age, apnoea/hypopnoea index and critical value of positive end-expiratory pressure (Pcrit) values. The mean CPAP level recommended in regular sleep studies for therapeutic purpose, available for nine of the patients, was $8.8 \pm 2.6 \mathrm{cmH}_{2} \mathrm{O}$. The corresponding lowest $P \mathrm{n}$ level that prevented flow limitation during propofol anaesthesia in these patients was $9.4 \pm 2.7 \mathrm{cmH}_{2} \mathrm{O}$ (not significant by paired t-test).

Figure 1 depicts the changes in GG-EMG and flow following the decrease in $P \mathrm{n}$ in one of the patients. At a $P \mathrm{n}$ of $10 \mathrm{cmH}_{2} \mathrm{O}$, there is no flow limitation. Lowering $P \mathrm{n}$ to $5 \mathrm{cmH}_{2} \mathrm{O}$ results in a typical flow pattern of flow limitation and flow continues to decrease gradually, stabilising at the third or fourth breath. At the same time, both tonic and phasic GG-EMG increases gradually. The EMG continues to increase while the flow signal remains stable and stabilises at about the ninth to tenth breath at a level of about $60 \%$ of maximum. In the mean, patients were maintained at $P \mathrm{n}$ of $5.8 \pm 2.5 \mathrm{cmH}_{2} \mathrm{O}$ and a similar response to the reduction of $P_{n}$ was observed in all.

Awakening from propofol anaesthesia was similar to arousal from sleep, accompanied by a large burst of dilator muscle EMG activity and body movements. It occurred 6-19 min after propofol infusion was stopped $(11.3 \pm 3.8 \mathrm{~min})$. Breathing instability and movements during awakening prevented adequate evaluation and comparison of data. Therefore, preawakening data were collected over the last stable breaths before emergence from anaesthesia, and post-awakening data

\begin{tabular}{|c|c|}
\hline TABLE 1 & $\begin{array}{l}\text { Anthropomorphic and sleep-study data of } \\
\text { patients }\end{array}$ \\
\hline AHI events $\cdot h^{-1}$ & $30.8 \pm 24.8(6--94)$ \\
\hline Age yrs & $46.5 \pm 11.1(29-70)$ \\
\hline BMI $\mathbf{k g} \cdot \mathrm{m}^{-2}$ & $29.5 \pm 3.5(24-36)$ \\
\hline Apnoeas \% & $35.4 \pm 29.7(2-90)$ \\
\hline Lowest $\mathrm{Sa}, \mathrm{O}_{2} \%$ & $83.1 \pm 7.5(66-91)$ \\
\hline Pcrit $^{\#} \mathrm{cmH}_{2} \mathrm{O}$ & $0.97 \pm 3.26(-6.2-5.8)$ \\
\hline
\end{tabular}

Data are presented as mean \pm SD (range). AHI: apnoea/hypopnoea index; BMI: body mass index; apnoeas \%: apnoeas as a percentage of apnoeas and hypopnoeas; $\mathrm{Sa}_{2} \mathrm{O}_{2}$ : arterial oxygen saturation; Pcrit: critical value of positive end-expiratory pressure; ${ }^{*}$ : obtained during anaesthesia. 


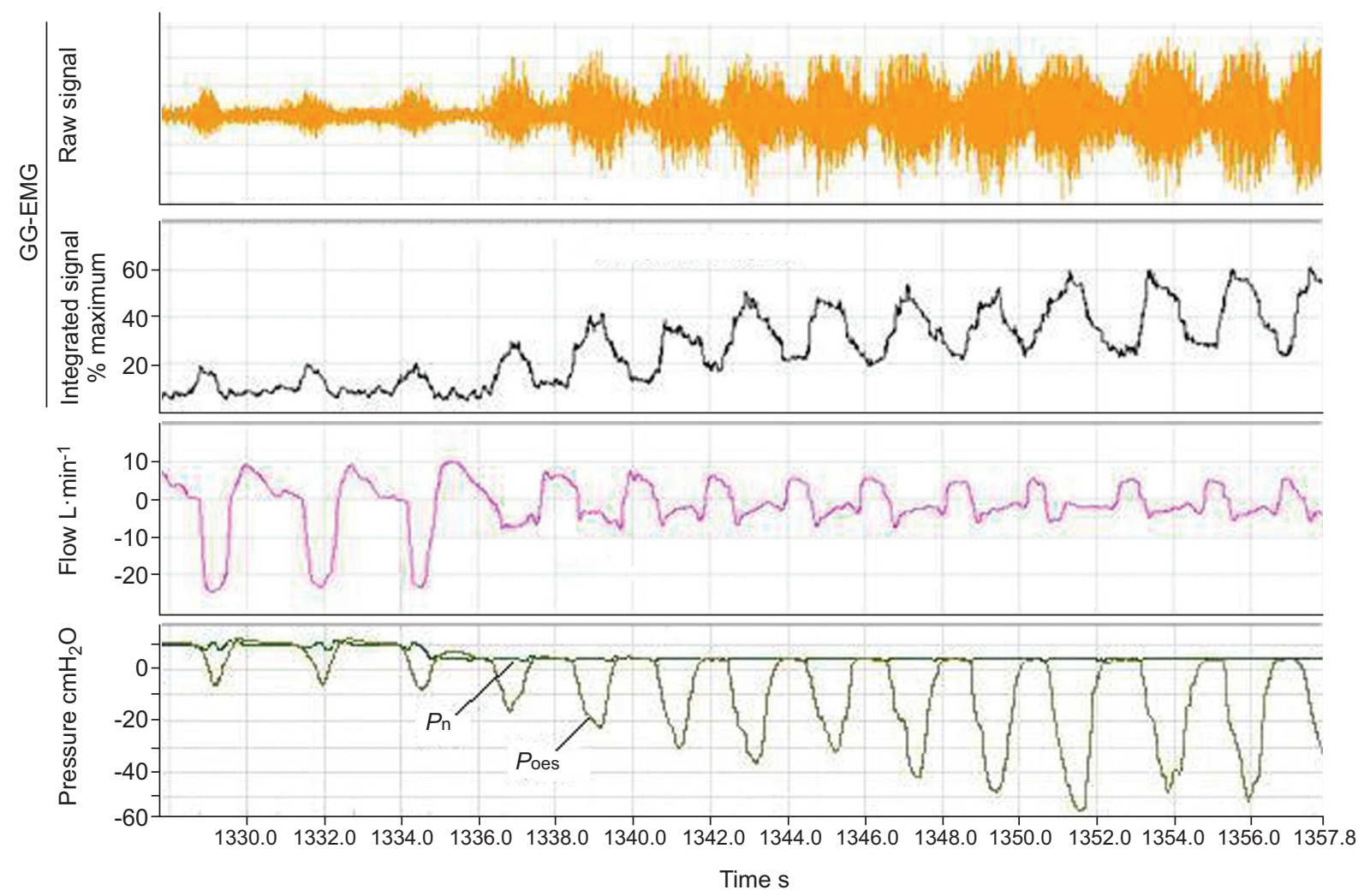

FIGURE 1. Changes in genioglossus (GG) electromyogram (EMG), flow and oesophageal pressure (Poes) following the decrease in continuous positive airway pressure in one of the patients. At nasal pressure $(P \mathrm{n})$ of $10 \mathrm{cmH}_{2} \mathrm{O}$, there is no flow limitation (inspiratory flow is recorded in the negative direction). Lowering $P_{\mathrm{n}}$ to $5 \mathrm{cmH} \mathrm{C}_{2} \mathrm{O}$ results in a typical flow pattern of flow limitation, and flow continues to decrease gradually, stabilising at the third or fourth breath. At the same time, both tonic and phasic GG-EMG increase gradually without any visible effect on flow. Increasing negative inspiratory deflections parallel increases in peak GG-EMG.

during the first stable breaths 1-3 min after awakening. Figure 2 compares tracings of one of the patients, including EMG, flow and oesophageal pressure, recorded shortly before and after awakening. Figure 3 depicts the corresponding intrapharyngeal pictures. Flow limitation and negative intrathoracic pressure fluctuations were associated with marked narrowing at the level of the velopharynx during inspiration, despite substantial activation of the GG and additional dilator muscles. Awakening resulted in enlargement of the pharynx, increase in flow and decrease in oesophageal pressure, followed by fast decrease in all muscles EMG. As seen in table 2, this pattern of response to awakening was seen in all patients, with awakening clearly changing the relationships between dilator muscle EMG and pharyngeal patency, i.e. the pharynx enlarges while the EMG decreases. Awakening prevented pharyngeal narrowing during inspiration, although a significant decrease in all muscles peak inspiratory EMG activity was found. Comparing the response of the GG to that of other dilator muscles in the subgroup of patients in whom additional muscles were evaluated $(n=14)$, changes in both tonic and inspiratory GG-EMG activity during the transition from anaesthesia to wakefulness were significantly larger than those of the other dilator muscles ( $\mathrm{p}<0.05$ for both). Also, in contrast to the GG, tonic activity of the other dilator muscles tended to remain unchanged, both during flow limitation and after awakening, and only their inspiratory activity increased gradually, and then decreased after awakening (table 3). Together, the tonic and peak inspiratory activity of the additional muscles evaluated changed from $11.6 \pm 6.1 \%$ to $13.0 \pm 6.3 \%$ of the maximum (not significant) and from $36.2 \pm 29.6 \%$ to $17.1 \pm 9.7 \%$ of the maximum $(p<0.05)$, respectively, before and after awakening. The difference between pre- and post-awakening EMG of the GG was significantly larger for the GG compared to all other muscles, both for tonic and inspiratory activity $(\mathrm{p}<0.05)$.

Electrical stimulation of the GG, assessed at the same $P_{n}$ level chosen for each of the individual patients after propofol administration was stopped, increased airflow, peak inspiratory and end-expiratory CSA significantly (fig. 4 and table 2), although, in the mean, it did not reach the levels observed after awakening.

\section{DISCUSSION}

In the present study we evaluated the EMG response of the GG and additional peri-pharyngeal muscles to flow limitation in OSA patients under mild propofol anaesthesia, and compared the effect of these changes on airflow and CSA at the area of collapse. The main findings of the study were: 1) introducing a steady-state hypopnoea-like flow limitation resulted in a gradual increase in dilator muscle EMG activity; 2) this increase in EMG failed to reduce pharyngeal obstruction, although electrical stimulation of the GG under the same conditions did enlarge the pharynx and increased airflow; 3) awakening was associated with immediate enlargement of the pharynx and restoration of normal airflow, associated with fast reduction in dilator muscle EMG activity. 
a)

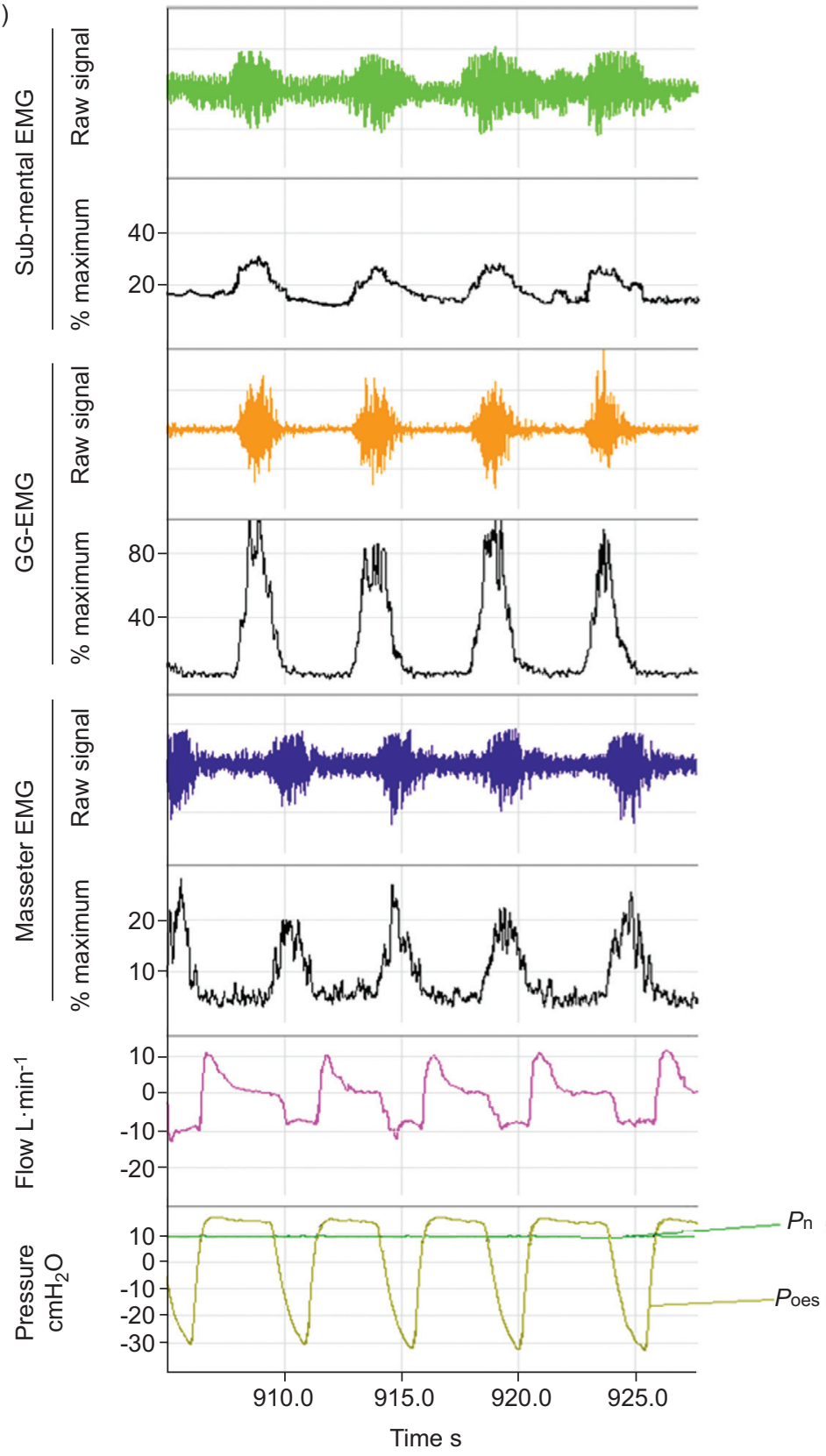

b)

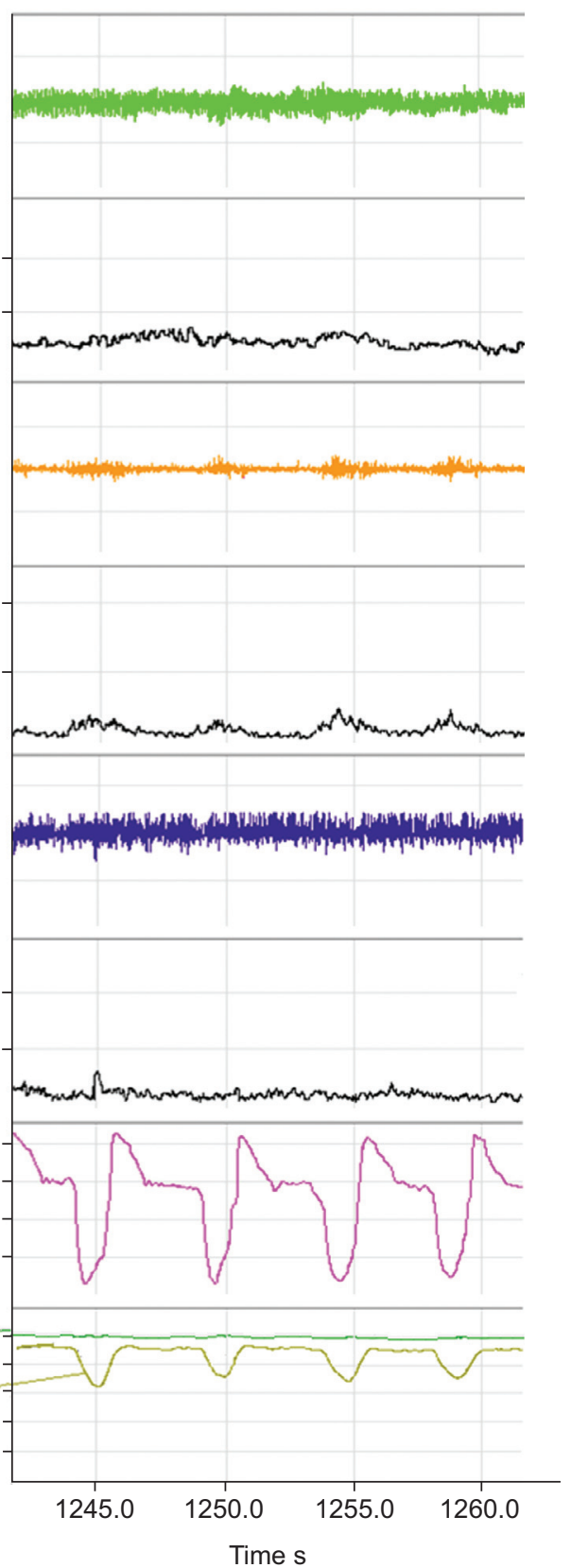

FIGURE 2. Tracings of one of the patients, including electromyogram (EMG), flow and oesophageal pressure (Poes), recorded shortly before and after awakening. Note a) the high EMG activity before awakening, particularly that of the genioglossus (GG), that reached $>80 \%$ of the maximum produced while awake, compared to b) the negligible EMG activity at rest after awakening, but normal flow without flow limitation in the latter. Pn: nasal pressure.

Our findings indicate that under mild propofol anaesthesia, the mechanical response to electrical stimulation of the GG is well maintained, while the response to central neural activation of the GG, as reflected by the EMG, seems to be modified and attenuated. We found, in all our patients, a gradual increase in dilator EMG activity following the introduction of flow limitation. This enhanced activity was probably mediated by intrapharyngeal and intrathoracic mechanoreceptors, as well as by chemoreceptors. Although hypoxaemia was prevented by supplementing oxygen whenever needed, in three of our first patients' ABG samples were drawn during progressive flow limitation, and carbon dioxide tension levels ranged between 48 and $52 \mathrm{mmHg}$. The gradual increase in dilator EMG activity had no effect on airflow. Even more impressive was the finding that the large increase in dilator activity during the continuous hypopnoea, which failed to restore pharyngeal patency, was followed by minimal activity after awakening, when pharyngeal patency was fully restored (fig. 2). This observation seems to provide sufficient evidence to suggest that the relationship between dilator muscle electrical activity and flow mechanics changes during the transition from propofol anaesthesia to wakefulness. 

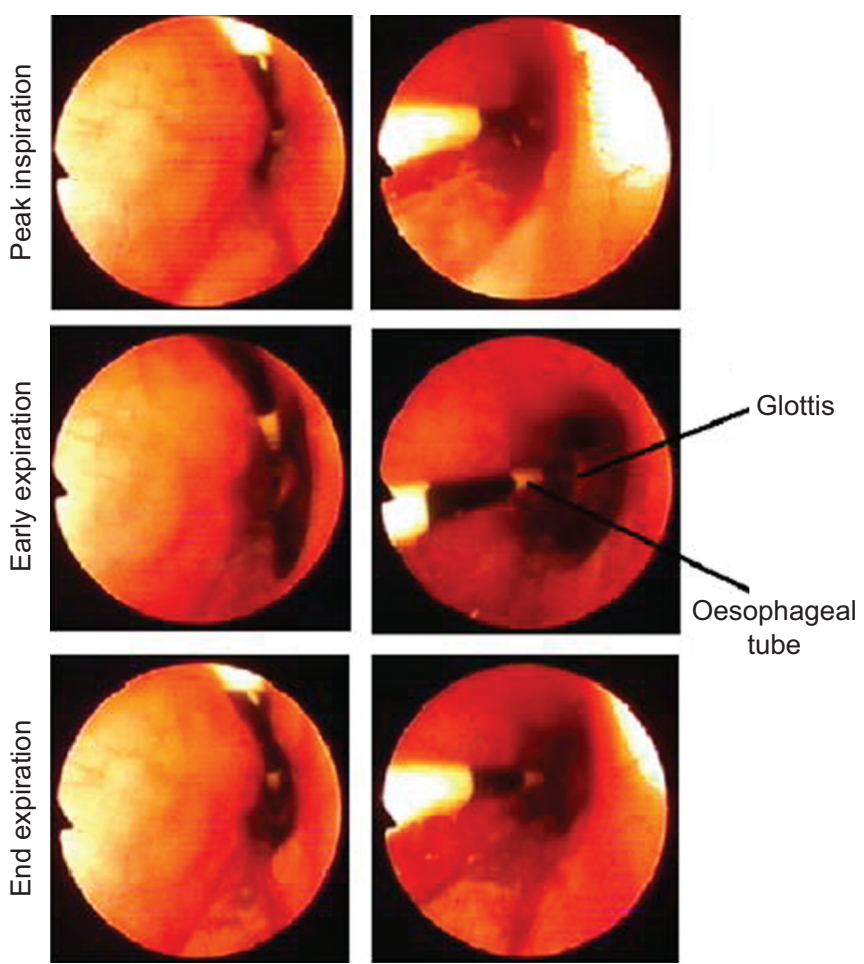

Pre-awakening

Post-awakening

FIGURE 3. Intrapharyngeal pictures corresponding to tracings in figure 2. Flow limitation and negative intrathoracic pressure fluctuations were due to marked narrowing at the level of the velopharynx during inspiration. Awakening resulted in immediate enlargement of the pharynx, also during expiration.

The human pharyngeal airway is surrounded by highly compliant soft tissues, resulting in collapse and complete obstruction not only in OSA patients, but in most people during anaesthesia or coma [6,7]. Therefore, active forces are required to maintain positive intraluminal pressure during inspiration and patency to airflow. Since the pioneering studies of REMMERs et al. [18], it is believed that the forces preventing pharyngeal obstruction are produced primarily by the upper airway dilator muscles. The reduction in GG-EMG activity with the onset of sleep $[8,9]$ and the diminution of the reflex response of the GG to negative pressure [19] and chemical drive [20] during sleep suggested that the increase in pharyngeal collapsibility during sleep is due primarily to sleep-associated decrease in dilator muscle activity.

However, it is well documented that upper airway dilator muscle EMG activity is restored later on during sleep and increases in response to respiratory stimulations, although usually with longer delay and to a lesser magnitude than during wakefulness [11]. Similarly, abundant observations demonstrated that dilator muscle EMG tends to increase in sleeping OSA patients during hypopnoeas and apnoeas, but this enhanced activity usually fails to restore pharyngeal patency before arousal $[4,15,18,21-24]$. The finding that increasing GG-EMG activity fails to affect airflow below an individual threshold could be demonstrated also during sleep [15]. In fact, the magnitude of inspiratory airflow appears independent of phasic GG activity both within any given flowlimited inspirations and across a series of flow-limited breaths.

The cause for the failure of the activated peri-pharyngeal muscles to produce an adequate dilatory and flow-mechanical action is poorly understood. By studying our patients under propofol sedation, arousal was prevented. It enabled uninterrupted increase in respiratory drive due to prolonged flow limitation, stimulating peak GG-EMG to levels $>60 \%$ of maximum, without any effect on airflow. This finding is particularly intriguing as the GG has been shown to be the most effective pharyngeal dilator $[25,26]$ and selective electrical stimulation of the GG with stimulation intensities that did not cause arousal were sufficient to improve airflow during sleep [16]. Conversely, after awakening, the pharynx enlarged and normal flow was restored, although GG-EMG activity declined to a minimum. Possible explanations for this phenomenon can be divided into two groups: 1) mechanical alterations occurring during anaesthesia may disrupt the mechanical response to dilator muscle contraction; and 2) anaesthesia alters the required coordination of muscles involved in the maintenance of pharyngeal patency.

Our findings can be described as indicating that anaesthesiainduced mechanical alterations raise the threshold dilator

TABLE 2 Cross-sectional area, flow and electromyogram (EMG) data immediately after lowering nasal pressure (baseline), during electrical stimulation (ES) of the genioglossus (GG) at the end of prolonged flow limitation, before awakening and after awakening

\begin{tabular}{|c|c|c|c|c|}
\hline & \multicolumn{3}{|c|}{ Anaesthesia } & \multirow[t]{2}{*}{ Awake } \\
\hline & Baseline & GG-ES & Pre-awake & \\
\hline \multicolumn{5}{|c|}{ Cross-sectional area $\mathrm{mm}^{2}$} \\
\hline End-expiration & $58.4 \pm 61.3$ & $85.4 \pm 61.3^{*}$ & $88.4 \pm 26.2$ & $134.9 \pm 126.9^{\#}$ \\
\hline Peak inspiration & $25.1 \pm 28.9$ & $66.3 \pm 75.5^{\star}$ & $11.8 \pm 8.2$ & $80.4 \pm 83.5^{\# \#}$ \\
\hline Flow L. $\min ^{-1}$ & $11.5 \pm 6.5$ & $18.6 \pm 9.2^{* \star \star}$ & $11.4 \pm 6.4$ & $25.1 \pm 8.3^{\# \# \#}$ \\
\hline \multicolumn{5}{|l|}{ GG-EMG \%max } \\
\hline Tonic & $4.4 \pm 3.8$ & & $15.7 \pm 12.2^{\star \star}$ & $9.1 \pm 6.1^{\#}$ \\
\hline Peak inspiration & $13.0 \pm 10.7$ & & $62.2 \pm 32.7^{\star \star \star}$ & $17.7 \pm 10.2^{\# \# \#}$ \\
\hline
\end{tabular}

Data are presented as mean \pm SD. ${ }^{*}: \mathrm{p}<0.05 ;{ }^{* *}: \mathrm{p}<0.01{ }^{* * *}: \mathrm{p}<0.001$ compared with baseline; ${ }^{\#}: \mathrm{p}<0.05 ;{ }^{\# \#}: \mathrm{p}<0.01 ;{ }^{\# \# \#}: \mathrm{p}<0.001$ compared with pre-awakening. 


\begin{tabular}{|c|c|c|c|c|c|}
\hline TABLE 3 & electrical & of the up & dilator muscles & $d$ in the pre & $y$, in addition to the \\
\hline \multirow[t]{2}{*}{ Muscle } & \multirow[t]{2}{*}{ Subjects $n$} & \multicolumn{2}{|c|}{ Anaesthesia } & \multicolumn{2}{|c|}{ Awake } \\
\hline & & Tonic & Peak inspiration & Tonic & Peak inspiration \\
\hline Geniohyoid & 2 & $5.0 \pm 2.8$ & $19.5 \pm 20.5$ & $7.0 \pm 5.7$ & $7.5 \pm 1.7$ \\
\hline Masseter & 4 & $14.5 \pm 0.7$ & $33.0 \pm 14.6$ & $15.0 \pm 5.7$ & $15.0 \pm 5.7$ \\
\hline Medial pterygoid & 4 & $11.5 \pm 9.3$ & $35.8 \pm 43.0$ & $14.3 \pm 10.3$ & $21.5 \pm 12.1$ \\
\hline
\end{tabular}

muscle activity has to surpass in order to enlarge the pharynx, to a level not reached under the experimental conditions. For example, positional changes that affect GG and/or other dilator muscles length during anaesthesia could alter their lengthtension relationship and reduce their contractile force. We have found in animal studies that maximal alterations in head and tracheal position reduce the effect and magnitude of shortening of dilator muscles [25, 27]. However, under physiological conditions, this effect in humans seems to be minimal [28], and we did not observe visible positional changes in pharyngeal structures associated with anaesthesia. Visco-elastic forces known to affect flow on the first breaths after lowering CPAP [14] could continue to counterbalance the increases in GG-EMG. Such forces are unlikely to continue for several minutes, and would not explain the fast mechanical recovery after awakening. Similarly, increased pharyngeal fluid lining surface tension that may reduce the dilatory efficacy of the GG is unlikely to decrease immediately following awakening. REMMERS et al. [18] explained this finding by failure of the dilators to counteract the large negative intraluminal pressures that develop downstream to the obstruction. This explanation disregards the fact that during pharyngeal obstruction, the inspiratory negative intrathoracic pressures develop in a ramp-like fashion, while the phasic GGEMG starts slightly before that of the diaphragm, usually with a steep burst $[12,29]$. Also, it does not contemplate an airway that is flow limited rather than completely occluded, when the increase in negative intraluminal pressures is relatively modest: in our patients, in the setting of flow limitation, oesophageal pressures were always above $-35 \mathrm{cmH}_{2} \mathrm{O}$. In fact, increasing negative downstream pressure during inspiration after the initiation of flow limitation tended to decrease inspiratory CSA, resulting in stable flow limitation (table 2). This effortindependent constant flow limitation is characteristic for the passive collapsible tube, as modelled by the Starling resistor [30]: according to this concept, once flow limitation occurs, the magnitude of flow through a collapsible tube is independent of downstream pressure, and is determined only by mechanical characteristics of the flow-limiting segment. Conversely, negative effort dependence, i.e. decrease in flow with lower downstream pressures, has been documented in partially obstructed OSA patients [31]. The cause of this negative effort dependence is unclear, but it is also predicted by our mathematical model, which characterises flow through a collapsible orifice [32]. As increases in inspiratory GG-EMG parallel the decreases in oesophageal pressure, it is possible that augmented GG activity is offset by the negative effort dependence, and the CSA of the flow limiting segment is determined by the balance between these two forces, i.e. a larger increase in GG-EMG due to augmented drive could have enlarged the pharynx. This argument fails to explain the development of large negative intraluminal pressures to begin with, as after a few partially obstructed breaths, both tonic and early-inspiratory dilator muscle EMG activity before each breath are already higher than during wakefulness (fig. 1). Also, end-expiratory CSA (i.e. in the absence of flow and negative intraluminal pressures) during anaesthesia was significantly smaller than after awakening, despite higher tonic GG-EMG activity (table 2). This finding also suggests that forces not related to GG-EMG determine pharyngeal patency during wakefulness and their effect is diminished during anaesthesia.

To assess whether the apparent inefficiency of dilator muscles is secondary to anaesthesia-induced mechanical alterations, we evaluated the response of the GG to electrical stimulation, in the same patients and equal $P$ n. The response to electrical stimulation was independent of downstream pressure (i.e. the same increase in flow was observed when stimulation was started before and during inspiration (fig. 4)), and indicated that the GG was not mechanically ineffective. It should be noted that we have previously found that the mechanical effect of GG stimulation during sleep and anaesthesia is similar [33]. Moreover, the site used for electrode placement in the current study produces a substantially smaller effect than a location that activates preferentially the protrusive GG fibres [34]. In addition, electrical stimulation produces a much smaller protrusive force than volitional activation [35]. All these considerations suggest that the mechanical effect of physiological activation of the dilator muscles, as represented by their EMG, is expected to exceed the magnitude observed with stimulation of the GG, as performed in this study. Nevertheless, the intensity of electrical stimulation exceeds that of the EMG substantially and current spread to adjacent muscles cannot be excluded. Accordingly, it is impossible to compare the intensity of neural and electrical activation of the GG, and it is possible that GG activity during electrical stimulation surpassed the threshold needed to enlarge the site of collapse.

The second possible explanation of our findings is that anaesthesia alters the coordination of muscles that maintain pharyngeal stability. This explanation also implies that during anaesthesia (and possibly also during sleep), GG-EMG does 

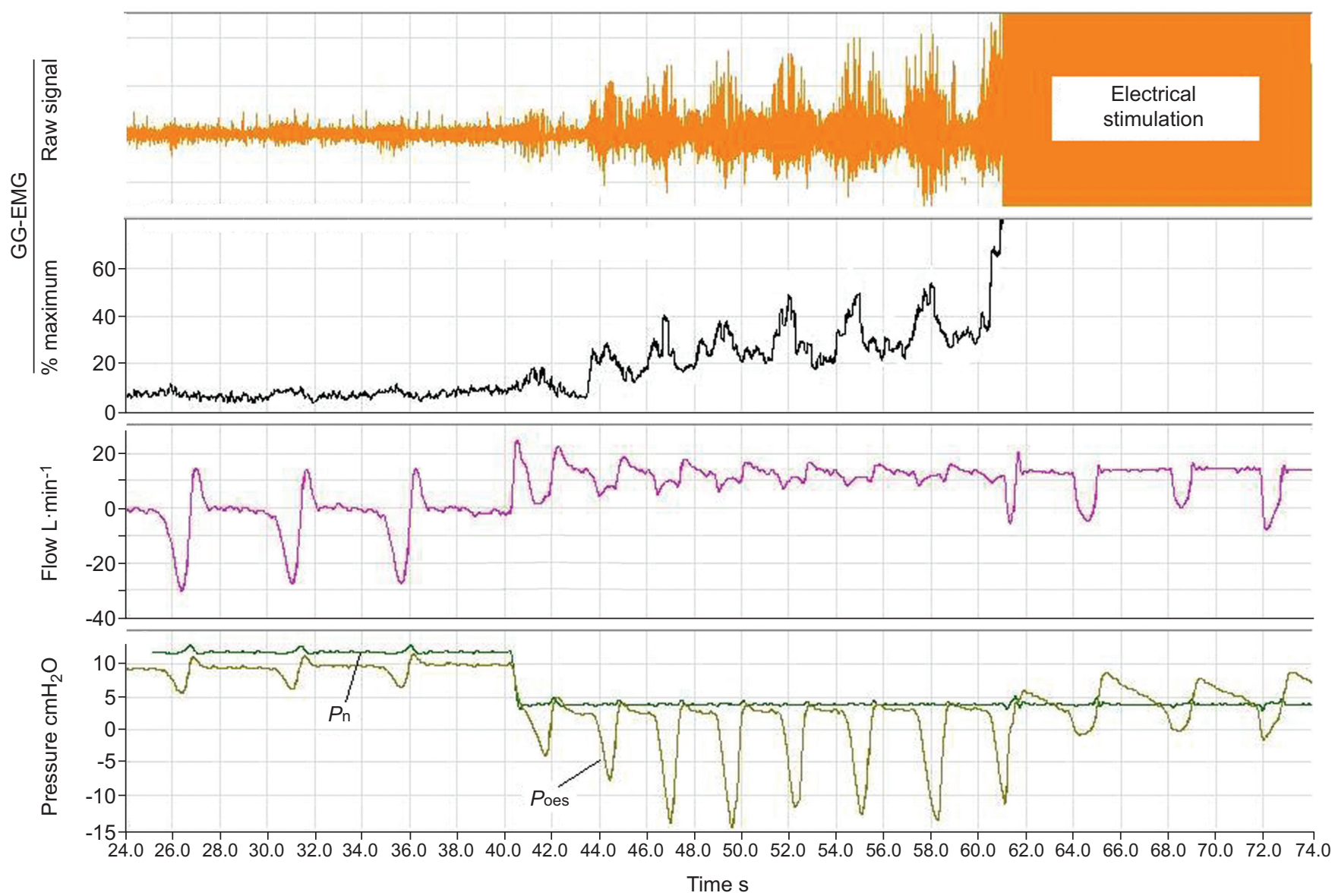

FIGURE 4. Flow response to electrical stimulation of the genioglossus (GG) muscle. Lowering nasal pressure $\left(P_{n}\right)$ caused progressive flow limitation. Electrical stimulation increased flow considerably. Note that the later-than-intended start of stimulation in this tracing enabled the GG electromyogram (EMG) to increase substantially, without any effect on flow. Also, stimulation started during inspiration, at high-negative oesophageal pressure (Poes), but this did not reduce the effect of GG contraction on flow. Due to the close proximity of the stimulation and recording electrodes, the stimulation artefact was always off-scale, even when very low and ineffective stimulation intensities were applied.

not represent the sum of dilatory forces that dilate the pharynx. The possibility that a sleep-induced imbalance in both the magnitude and timing of electrical activity between the upper airway and chest wall inspiratory muscles plays a role in the pathogenesis of sleep apnoea was raised long ago [36, 37]. Similarly, the muscular-hydrostat model of tongue function proposes a constant coordinated interaction of extrinsic and intrinsic tongue muscles in all tongue movements [38]. Accordingly, it has been proposed that pharyngeal obstruction may be secondary to failure of other dilator muscles to act similar to the GG $[11,39]$. To address this possibility, we evaluated the EMG response of several dilator muscles, but could not find signs of un-coordination. Nevertheless, lack of adequate activation of muscles not evaluated in this study cannot be excluded. Anaesthesia-induced general hypotonia that includes the trunk and neck muscles may affect the forces arising from the chest and increase extrapharyngeal pressure within the maxillo-mandibular bony structures that enclose the soft tissue surrounding the collapsible segment of the pharynx $[34,40]$. Such alterations may affect GG contraction and/or the negative dependence of flow and downstream pressure. Coordinated co-activation of retractor muscles, not evaluated in the present study, may be important for the mechanical response [41]. In addition, it is conceivable that excessive activation of pharyngeal constrictors could prevent pharyngeal dilatation by the GG. However, pharyngeal constrictors has been shown to improve pharyngeal stability by KUNA [42], and that author's finding in that study that the constrictors may have different effects on airway patency depending on luminal size lends support to the idea that combinations of muscle activity determine the state of pharyngeal patency, rather than the activity of any one muscle by itself. It should be noted that all muscles evaluated in the present study may be mechanically irrelevant for the velopharynx, which was the site of obstruction in all our patients. This possibility cannot be fully rejected by the preserved response to electrical stimulation of the GG in the same patients and the same condition and we did not evaluate palate muscle EMG. Accordingly, it is possible that augmented drive to the GG may be more effective in patients with oropharyngeal sites of obstruction. In addition to intermuscular coordination, intramuscular coordination of fibre activity may also be important. Although the GG is considered to be a single muscle, its fan-like fibre orientation results in different mechanical activity depending on the fibres recruited [34]. Recent studies that have evaluated single motor-units of the GG have shown that this muscle's EMG is composed of several 
populations of units that are activated differently during breathing $[29,43]$. These motor units are coordinated by complex premotor networks $[29,44]$ that may be affected by anaesthesia [45]. The activity of different GG motor units changes differently during the transition from wakefulness to sleep [46-48]. Accordingly, the response of the GG to the transitions between sleep and wakefulness includes reorganisation of the pattern of motor unit activation within the muscle. It has previously been demonstrated in a canine preparation that dilator muscle activation, although closely paralleling inspiration-associated pharyngeal enlargement, was not the sole cause of this enlargement, as phasic enlargements continued after dissection of the nerves to the dilator muscles [49]. This finding led to the recognition of the importance of forces arising in the thorax on pharyngeal patency, and was confirmed in a later study in decerebrated cats, which demonstrated that transection of the hypoglossus nerve did not affect Pcrit [50]. In more recent human studies, carbon dioxide-induced augmentation in GG-EMG produced only minor improvement in pharyngeal stability in OSA patients, as compared to changes in lung volume [51]. All these considerations suggest that anaesthesia-induced changes in activation and/or coordination of muscles involved in the maintenance of pharyngeal patency may explain the observed lack of flow response to the increase in GG-EMG. Nevertheless, this possibility remains speculative, as our study did not provide evidence for its occurrence, while demonstrating an increase in activity of the dilator muscles evaluated. In addition, distinguishing mechanical from control mechanisms is arbitrary, as both are often interdependent.

Several limitations of this study and potential confounders have to be acknowledged. First, this is a laboratory-based study, evaluating physiological parameters under propofol anaesthesia. We choose to use anaesthesia to assess the mechanical effect of electrical stimulation, to enable endoscopic evaluation and to allow prolonged hypopnoeas without interruption by arousal, tasks that could not be performed during normal sleep. Propofol anaesthesia is often referred to as chemical- or drug-induced sleep $[45,52]$. Although propofol induces a state of coma that can be classified by EEG from minimally conscious state (phase 1) to full coma resembling brain death (phase 4), under mild anaesthesia, motor tone and respiration are preserved [53]. Propofol does not have a direct effect on striated muscle [54, 55], but its effect on upper airway dilator muscles has not been specifically evaluated. Spontaneous breathing is the harbinger of recovery from anaesthesia, and integrates both muscular activity and active respiratory control network in the ventral medulla and pons [45]. Hence, control of motor tone and respiratory activity should not be fundamentally different between light anaesthesia and sleep. Also, the anaesthetic effects of propofol are mediated by its effects on monoaminergic, $\gamma$-aminobutyric acid-ergic, glutamatergic, cholinergic and adenosinergic neurotransmission in brain regions known to regulate sleep and wakefulness [56] and EEG patterns during anaesthesia resembles those seen in slow-wave sleep [45]. The loss of connectivity between different brain regions is also similar during sleep and anaesthesia [57]. Our patients were only lightly anesthetised, as indicated by the presence of spontaneous breathing and intensive respiratory response to flow limitation throughout the experiment, and the level of sedation further diminished before the emergence from anaesthesia. We have recently found that, by manipulating CPAP levels in a way that prolonged the duration of hypopnoeas, marked increases in GG-EMG could be achieved in OSA patients during sleep. Several-fold increases in EMG activity during sleep over wakefulness, despite equal negative intrathoracic pressures, did not cause any improvement in airflow [58]. Based on these findings and the above considerations, we believe that propofol anaesthesia merely unmasked and augmented, by delaying arousal, the magnitude of the electro-mechanical dissociation present also during sleep. Nevertheless, pharmacological anaesthesia and sleep are not identical and differ in terms of both neurophysiology and neurochemistry [56]. Also, in contrast to anaesthesia, pharyngeal patency can sometimes be restored during sleep without EEG or other signs of arousal [15]. Therefore, all findings and conclusions of the present study are limited to propofol anaesthesia, and the relevance of our findings to the state of sleep remains to be demonstrated. Secondly, the GG-EMG was recorded from one site of the muscle (retromandibular) and may only partially represent the whole GG. Thirdly, only a few dilator muscles were evaluated. Therefore, the response of these muscles represents primarily a qualitative finding, indicating the presence of increased drive also to these muscles. Also, sealing of the mouth undertaken to prevent mandibular movements and air leaks could impede tongue mobility in response to neural activation. Finally, the mean increase in peak inspiratory GG-EMG to $\sim 60 \%$ of maximum appears rather high. It may suggest that our measurements of maximal GG-EMG, which are voluntary manoeuvres that depend on patients' cooperation and were performed after propofol anaesthesia, may have underestimated the true maximal GG-EMG. However, the mean value of GG-EMG during wakefulness in our patients, presented as \% maximum, was substantially lower than that reported by MezzanotTe et al. [59]. The tongue protrusion manoeuvres undertaken to assess maximal GG-EMG were performed 20-30 min after awakening, when the patients were fully awake and cooperative; at least three reproducible levels were obtained, and we were confident that the patients made their best effort. To confirm that the proximity to anaesthesia had no effect on the level of maximal effort the patients performed, we compared maximal volitional arm flexion force measured in the supine position with a strain gauge, before and after propofol anaesthesia, in five subjects that underwent minor surgical procedures. Maximal arm flexion measured before and 10-15 min after awakening, when patients appeared to be awake and fully alert, were $14.7 \pm 2.4$ and $13.9 \pm 2.9 \mathrm{~kg}$, respectively, with two subjects producing higher forces after anaesthesia. More importantly, presentation of EMG as percent of maximum was undertaken mainly to provide a denominator enabling to present group mean EMG data. All comparisons were paired, and the wide dissociation between EMG and mechanics when comparing anaesthesia and wakefulness was observed in every patient. Therefore, underestimation of the maximal EMG would not alter our main finding: with the initiation of hypopnoeas GG-EMG increased dramatically without any effect on flow, with the pharynx remaining narrow and obstructed, while after awakening the pharynx was patent in the presence of low GG-EMG activity.

In conclusion, our findings indicate that in propofol-anaesthetised OSA patients, as previously shown in anesthetised animals 
$[49,50]$, dilator muscle electrical activity may represent neural drive to the specific muscle, but has no direct relationship to pharyngeal patency. The cause of this increase in EMG activity with no improvement in airflow during anaesthesia, and increased airflow upon awakening with reductions of EMG activity, are puzzling and unresolved. Nevertheless, these observations suggest that other forces, probably forces arising in the thorax concomitantly with upper airway muscle activation and affecting upper airway mechanics, are more important for pharyngeal stability and patency under anaesthesia, as demonstrated during sleep $[51,60]$. If the altered mechanical efficiency of augmented dilator muscle activity occurs also during sleep, it may suggest that fluctuations in the propensity to respiratory disturbances in OSA patients over night can be attributed, at least in part, to such alterations, rather than to changes in the intensity of activation of these muscles, although the latter are likely to be also important $[15,61]$. The causative mechanism(s) responsible for the failure of dilator muscle contraction to maintain pharyngeal patency may represent the "wakefulness stimulus" [9] that distinguish control of pharyngeal patency during wakefulness and sleep.

\section{STATEMENT OF INTEREST}

None declared.

\section{REFERENCES}

1 Malhotra A, Fogel RB, Edwards JK, et al. Local mechanisms drive genioglossus activation in obstructive sleep apnea. Am J Respir Crit Care Med 2000; 161: 1746-1749.

2 White DP. Pathogenesis of obstructive and central sleep apnea. Am J Respir Crit Care Med 2005; 172: 1363-1370.

3 Younes M. Contributions of upper airway mechanics and control mechanisms to severity of obstructive apnea. Am J Respir Crit Care Med 2003; 168: 645-658.

4 Patil SP, Schneider H, Marx JJ, et al. Neuromechanical control of upper airway patency during sleep. J Appl Physiol 2007; 102: 547-556.

5 Eastwood PR, Platt PR, Shepherd K, et al. Collapsibility of the upper airway at different concentrations of propofol anesthesia. Anesthesiology 2005; 103: 453-454.

6 Oliven A, Aspandiarov E, Gankin I, et al. Collapsibility of the relaxed pharynx and risk of sleep apnoea. Eur Respir J 2008; 32: 1309-1315.

7 Safar P, Escarraga LS, Chang F. Upper airway obstruction in the unconscious patient. J Appl Physiol 1959; 14: 760-764.

8 Sauerland EK, Harper RM. The human tongue during sleep, electromyographic activity of the genioglossus muscle. Exp Neurol 1976; 51: 160-170.

9 Fogel RB, Trinder J, White DP, et al. The effect of sleep onset on upper airway muscle activity in patients with sleep apnoea versus controls. J Physiol 2005; 15, 564: 549-562.

10 Luo YM, Wu HD, Tang J, et al. Neural respiratory drive during apnoeic events in obstructive sleep apnoea. Eur Respir J 2008; 31: 650-657.

11 Younes M. Role of respiratory control mechanisms in the pathogenesis of obstructive sleep disorders. J Appl Physiol 2008; 105: 1389-1405.

12 Strohl KP, Hensley MJ, Hallett M, et al. Activation of upper airway muscles before onset of inspiration in normal humans. J Appl Physiol 1980; 49: 638-642.

13 Van Lunteren E, Strohl KP. The muscles of the upper airways. Clin Chest Med 1986; 7: 171-188.
14 Schwartz AR, O'Donnell CP, Baron J, et al. The hypotonic upper airway in obstructive sleep apnea. Role of structures and neuromuscular activity. Am J Respir Crit Care Med 1998; 157: 1051-1057.

15 Younes M, Andrea HS, Loewen ASH, et al. Genioglossus activity available via non-arousal mechanisms vs. that required for opening the airway in obstructive apnea patients. J Appl Physiol 2012; 112: 249-258.

16 Oliven A, O'Hearn DJ, Boudewyns A, et al. Upper airway response to electrical stimulation of the genioglossus in obstructive sleep apnea. J Appl Physiol 2003; 95: 2023-2029.

17 Oliven A, Tov N, Geitini L, et al. Effect of genioglossus contraction on pharyngeal lumen and airflow in sleep apnoea patients. Eur Respir J 2007; 30: 748-758.

18 Remmers JE, deGroot WJ, Sauerland EK, et al. Pathogenesis of upper airway occlusion during sleep. J Appl Physiol 1978; 44: 931-938.

19 Eckert DJ, McEvoy RD, George KE, et al. Genioglossus reflex inhibition to upper-airway negative-pressure stimuli during wakefulness and sleep in healthy males. J Physiol 2007; 581: 1193-1205.

20 Pillar G, Malhotra A, Fogel RB, et al. Upper airway muscle responsiveness to rising $\mathrm{PCO}_{2}$ during NREM sleep. J Appl Physiol 2000; 89: 1275-1282.

21 McGinley BM, Schwartz AR, Schneider $H$, et al. Upper airway neuromuscular compensation during sleep is defective in obstructive sleep apnea. J Appl Physiol 2008; 105: 197-205.

22 Berry RB, McNellis MI, Kouchi K, et al. Upper airway anesthesia reduces phasic genioglossus activity during sleep apnea. Am J Respir Crit Care Med 1997; 156: 127-132.

23 Okabe S, Chonan T, Hida W, et al. Role of chemical drive in recruiting upper airway and inspiratory intercostals intercostal muscles in patients with obstructive sleep apnea. Am Rev Respir Dis 1993; 147: 190-195.

24 Onal E, Lopata M, O'Connor TD. Diaphragmatic and genioglossal electromyogram responses to $\mathrm{CO}_{2}$ rebreathing in humans. J Appl Physiol 1980; 50: 1052-1055.

25 Odeh M, Schnall R, Gavriely N, et al. Dependency of upper airway patency on head position: the effect of muscle contraction. Respir Physiol 1995; 100: 239-244.

26 Schnall R, Pillar G, Kelsen SG, et al. Dilatory effects of upper airway muscle contraction induced by electrical stimulation in awake humans. J Appl Physiol 1995; 78: 1950-1956.

27 Oliven A, Odeh M. Effect of positional changes of anatomical structures on upper airway dilating muscle shortening during electro- and chemostimulation. J Appl Physiol 2006; 101: 745-751.

28 Oliven $\mathrm{R}$, Tov N, Odeh $\mathrm{M}$, et al. Interacting effects of genioglossus stimulation and mandibular advancement in sleep apnea. J Appl Physiol 2009; 106: 1668-1673.

29 Saboisky JP, Butler JE, Fogel RB, et al. Tonic and phasic respiratory drives to human genioglossus motoneurons during breathing. J Neurophysiol 2006; 95: 2213-2221.

30 Gold AR, Schwartz AR. The pharyngeal critical pressure (Pcrit). The whys and hows of using nasal continuous positive airway pressure diagnostically. Chest 1996; 110: 1077-1087.

31 Isono S, Feroah TR, Hajduk EA, et al. Interaction of cross sectional area, driving pressure, and airflow of passive velopharynx. J Appl Physiol 1997; 83: 851-859.

32 Oliven A, Kaufman E, Kaynan R, et al. Mechanical parameters determining pharyngeal collapsibility in patients with sleep apnea. J Appl Physiol 2010; 109: 1037-1044.

33 Oliven $\mathrm{A}$, Tov $\mathrm{N}$, Odeh $\mathrm{M}$, et al. Electrical stimulation of the genioglossus to improve pharyngeal patency in obstructive sleep apnea: comparison of results obtained during sleep and anesthesia. Harefuah 2009; 148: 157-161.

34 Dotan Y, Golibroda T, Oliven R, et al. Parameters affecting pharyngeal response to genioglossus stimulation in sleep apnoea. Eur Respir J 2011; 38: 338-347. 
35 Tov N, Odeh M, Weiman $\mathrm{Y}$, et al. Force and endurance of the tongue protrusors in patients with obstructive sleep apnea (OSA): volitional and electrophysiological properties. Proc Am Thorac Soc 2006; A314.

36 Hudgel DW. Differential activation of respiratory muscles during wakefulness and sleep. Prog Clin Biol Res 1990; 345: 233-242.

37 Hudgel DW, Harasick T. Fluctuation in timing of upper airway and chest wall inspiratory muscle activity in obstructive sleep apnea. J Appl Physiol 1990; 69: 443-450.

38 Kier WM, Smith KK. Tongues, tentacles and trunks: the biomechanics and movement of muscular hydrostats. Zool J Linn Soc 1985; 83: 207-324.

39 Jordan AS, White DP. Pharyngeal motor control and the pathogenesis of obstructive sleep apnea. Respir Physiol Neurobiol 2008; 160: 1-7.

40 Tsuiki S, Isono S, Ishikawa T, et al. Anatomical balance of the upper airway and obstructive sleep apnea. Anesthesiology 2008; 108: 1009-1015.

41 Oliven A, Odeh M, Geitini L, et al. Effect of coactivation of tongue protrusor and retractor muscles on pharyngeal lumen and airflow in sleep apnea patients. J Appl Physiol 2007; 103: 1662-1668.

42 Kuna ST. Respiratory-related activation and mechanical effects of the pharyngeal constrictor muscles. Respir Physiol 2000; 119: 155-161.

43 Saboisky JP, Butler JE, McKenzie DK, et al. Neural drive to human genioglossus in obstructive sleep apnoea. J Physiol 2007; 582: 135-146.

44 Laine CM, Bailey EF. Common synaptic input to the human hypoglossal motor nucleus. J Neurophysiol 2011; 105: 380-387.

45 Brown EN, Lydic R, Schiff ND. General anesthesia, sleep, and coma. N Engl J Med 2010; 363: 2638-2650.

46 Bailey EF, Fridel KW, Rice AD. Sleep/wake firing patterns of human genioglossus motor units. J Neurophysiol 2007; 98: 3284-3291.

47 Wilkinson V, Malhotra A, Nicholas CL, et al. Discharge patterns of human genioglossus motor units during sleep onset. Sleep 2008; 31 : 525-533.

48 Wilkinson V, Malhotra A, Nicholas CL, et al. Discharge patterns of human genioglossus motor units during arousal from sleep. Sleep 2010; 33: 379-387.
49 Van de Graaff WB. Thoracic influence on upper airway patency. J Appl Physiol 1988; 65: 2124-2131.

50 Rowley JA, Williams BC, Smith PL, et al. Neuromuscular activity and upper airway collapsibility: mechanisms of action in the decerebrated cat. Am J Respir Crit Care Med 1997; 156: 515-521.

51 Jordan AS, White DP, Owens RL, et al. The effect of increased genioglossus activity and end-expiratory lung volume on pharyngeal collapse. J Appl Physiol 2010; 109: 469-475.

52 Boudewyns A, Marklund M, Hochbab W. Alternatives for OSAHS treatment: selection of patients for upper airway surgery and oral appliances. Eur Respir Rev 2007; 106: 132-145.

53 Bevan JC, Veall GR, Macnab AJ, et al. Midazolam premedication delays recovery after propofol without modifying involuntary movements. Anesth Analg 1997; 85: 50-54.

54 Ginz HF, Zorzato F, Iaizzo PA, et al. Effect of three anaesthetic techniques on isometric skeletal muscle strength. Br J Anaesth 2004; 92: 367-372.

55 Nishina K, Mikawa K, Kodama S, et al. The effects of enflurane, isoflurane, and intravenous anesthetics on rat diaphragmatic function and fatigability. Anesth Analg 2003; 96: 1674-1678.

56 Van Dort CJ, Baghdoyan HA, Lydic R. Neurochemical modulators of sleep and anesthetic states. Int Anesthesiol Clin 2008; 46: 75-104.

57 Ferrarellia F, Massiminib M, Sarassoa S, et al. Breakdown in cortical effective connectivity during midazolam-induced loss of consciousness. Proc Natl Acad Sci 2010; 107: 2681-2686.

58 Dotan Y, Pillar G, Gaitini L, et al. Sleep-induced dissociation between neural drive and mechanical response of the genioglossus in obstructive sleep apnea. Am J Resp Crit Care Med 2012; 185: A5369.

59 Mezzanotte WS, Tangel DJ, White DP. Waking genioglossal electromyogram in sleep apnea patients versus normal controls (a neuromuscular compensatory mechanism). J Clin Invest 1992; 89: 1571-1579.

60 Squier SB, Patil SP, Schneider H, et al. Effect of end-expiratory lung volume on upper airway collapsibility in sleeping men and women. J Appl Physiol 2010; 109: 977-985.

61 Jordan AS, Wellman A, Heinzer RC, et al. Mechanisms used to restore ventilation after partial upper airway collapse during sleep in humans. Thorax 2007; 62: 861-867. 